Correction

\title{
Correction: He, X.; et al. Wireless Power Transfer System for Rotary Parts Telemetry of Gas Turbine Engine. Electronics 2018, 7(5), 58
}

\author{
Xiaoming $\mathrm{He}^{\dagger}$, Wenjun Shu ${ }^{+} \mathbb{D}$, Bing $\mathrm{Yu}^{*}$ (D) and Xiaodong Ma \\ Jiangsu Province Key Laboratory of Aerospace Power System, Nanjing University of Aeronautics and \\ Astronautics, Nanjing 210016, China; vehicle@nuaa.edu.cn (X.H.); shushushu@nuaa.edu.cn (W.S.); \\ mxd@nuaa.edu.cn (X.M.) \\ * Correspondence: yb203@nuaa.edu.cn; Tel.: +86-025-8489-2200 \\ + These authors contributed equally to this work.
}

Received: 4 June 2019; Accepted: 5 June 2019; Published: 25 June 2019

The authors wish to make the following corrections to the published paper [1].

There is a misprint in Equation (13), which expresses the mutual inductance $M$ between two coils. The term $2 d_{1} d_{2}$ is incorrect and should be replaced with $\mu_{0} d_{1} d_{2}$, where $\mu_{0}$ is the permeability of vacuum.

In summary, on page 6, Equation (13) should be changed from

$$
\begin{gathered}
M=\frac{2 d_{1} d_{2}}{4 \pi R \sqrt{d_{1}^{2}+d_{2}^{2}+h^{2}+x^{2}}} \int_{\theta_{2}=0}^{2 \pi} \int_{\theta_{1}=0}^{2 \pi} \cos \left(\theta_{1}-\theta_{2}\right) \times\left[1-\left(\alpha \cos \left(\theta_{1}-\theta_{2}\right)+\beta \cos \theta_{1}\right.\right. \\
\left.\left.-\delta \cos \theta_{2}\right)\right]-1 / 2 d \theta_{1} d \theta_{2}
\end{gathered}
$$

to the following correct version:

$$
\begin{gathered}
M=\frac{\mu_{0} d_{1} d_{2}}{4 \pi R \sqrt{d_{1}^{2}+d_{2}^{2}+h^{2}+x^{2}}} \int_{\theta_{2}=0}^{2 \pi} \int_{\theta_{1}=0}^{2 \pi} \cos \left(\theta_{1}-\theta_{2}\right) \times\left[1-\left(\alpha \cos \left(\theta_{1}-\theta_{2}\right)+\beta \cos \theta_{1}\right.\right. \\
\left.\left.-\delta \cos \theta_{2}\right)\right]-1 / 2 d \theta_{1} d \theta_{2}
\end{gathered}
$$

There is a misprint in Equation (14), which expresses the mutual inductance $M$ between two coils. The term $2 d_{1} d_{2}$ is incorrect and should be replaced with $\mu_{0} d_{1} d_{2}$, where $\mu_{0}$ is the permeability of vacuum.

In summary, on page 6, Equation (14) should be changed from

$$
M=\frac{2 d_{1} d_{2}}{4 \pi R \sqrt{d_{1}^{2}+d_{2}^{2}+h^{2}}} \int_{\theta_{2}=0}^{2 \pi} \int_{\theta_{1}=0}^{2 \pi} \cos \left(\theta_{1}-\theta_{2}\right)\left[1-\alpha \cos \left(\theta_{1}-\theta_{2}\right)\right]^{-1 / 2} d \theta_{1} d \theta_{2}
$$

to the following correct version:

$$
M=\frac{\mu_{0} d_{1} d_{2}}{4 \pi R \sqrt{d_{1}^{2}+d_{2}^{2}+h^{2}}} \int_{\theta_{2}=0}^{2 \pi} \int_{\theta_{1}=0}^{2 \pi} \cos \left(\theta_{1}-\theta_{2}\right)\left[1-\alpha \cos \left(\theta_{1}-\theta_{2}\right)\right]^{-1 / 2} d \theta_{1} d \theta_{2}
$$

There is a misprint in Equation (16), which expresses the mutual inductance $M$ between two coils. The term $2 d_{1, i} d_{2, j}$ is incorrect and should be replaced with $\mu_{0} d_{1} d_{2}$, where $\mu_{0}$ is the permeability of vacuum.

In summary, on page 6, Equation (16) should be changed from

$$
\begin{gathered}
M=\frac{2 d_{1, i} d_{2, j}}{4 R \sqrt{d_{1, i^{2}+d_{2, j}{ }^{2}+h^{2}+x^{2}}}} \int_{\theta_{2}=0}^{2 \pi} \int_{\theta_{1}=0}^{2 \pi} \cos \left(\theta_{1}-\theta_{2}\right) \times\left[1-\left(\alpha \cos \left(\theta_{1}-\theta_{2}\right)\right.\right. \\
\left.\left.+\beta \cos \theta_{1}-\delta \cos \theta_{2}\right)\right]-1 / 2 d \theta_{1} d \theta_{2}
\end{gathered}
$$


to the following correct version:

$$
\begin{aligned}
& M=\frac{\mu_{0} d_{1, i} d_{2, j}}{4 \pi R \sqrt{d_{1, i^{2}}+d_{2, j^{2}}+h^{2}+x^{2}}} \int_{\theta_{2}=0}^{2 \pi} \int_{\theta_{1}=0}^{2 \pi} \cos \left(\theta_{1}-\theta_{2}\right) \times\left[1-\left(\alpha \cos \left(\theta_{1}-\theta_{2}\right)+\beta \cos \theta_{1}\right.\right. \\
& \left.\left.-\delta \cos \theta_{2}\right)\right]-1 / 2 d \theta_{1} d \theta_{2}
\end{aligned}
$$

The authors would like to apologize for any inconvenience caused to the readers by these changes. The change does not affect the scientific results. The manuscript will be updated and the original will remain online on the article webpage, with a reference to this Correction.

Conflicts of Interest: The authors declare no conflict of interest.

\section{References}

1. He, X.; Shu, W.; Yu, B.; Ma, X. Wireless Power Transfer System for Rotary Parts Telemetry of Gas Turbine Engine. Electronics 2018, 7, 58. [CrossRef]

(C) 2019 by the authors. Licensee MDPI, Basel, Switzerland. This article is an open access article distributed under the terms and conditions of the Creative Commons Attribution (CC BY) license (http://creativecommons.org/licenses/by/4.0/). 